\title{
Prenatal and Postnatal Exposures to 1-Methyl-4-phenyl-1,2,3,6-tetra Hydropyridine (MPTP) Impaired Mouse Midbrain Dopamine System and May Produce a Predisposing and Inducing Model for Parkinson's Disease
}

\author{
Gladson Muthian, Jennifer King, Lemuel Dent, Marquitta Smith, Veronica Mackey, \\ Clivel Charlton \\ Department of Neuroscience and Pharmacology, Meharry Medical College, Nashville, USA \\ Email: ccharlton@mmc.edu
}

Received August 3, 2012; revised September 7, 2012; accepted September 21, 2012

\begin{abstract}
Dopamine cell bodies in the substantia nigra of the midbrain and with their terminals projecting to the neostriatum form the nigrostriatum and these dopamine neurons degenerate in Parkinson's disease (PD). Based on metabolic and functional specialization of the cell bodies versus the axon terminals, the level and disposition of dopamine, its metabolites and enzymes are different in both regions and are likely to be affected differently in PD. We examined changes in the midbrain dopamine system following 1-methyl-4-phenyl-1,2,3,6-tetrahydropyridine (MPTP), to test the hypothesis that a predisposing/sensitization stage and a inducing/precipitating stage underlie PD. Pregnant mice were treated with a low dose of MPTP during gestation days $8-12$ to model the predisposing/sensitization stage, by interrupting the fetal midbrain dopamine system during its neurogenesis. For the inducing/precipitating stage, the 12-week offspring were administered MPTP. The prenatal-MPTP offspring appear normal, but midbrain dopamine, 3,4-di-hydroxy-phenyl-aceticacid, 3-methoxytyramine, tyrosine-hydroxylase and L-aromatic-amino-acid-decarboxylase, were reduced by $49.6 \%$, $48 \%, 54 \%, 20.9 \%$ and $25 \%$. Postnatal-MPTP of $10,20,30 \mathrm{mg} / \mathrm{kg}$ administered to the prenatal-PBS vs prenatal-MPTP offspring reduced midbrain dopamine by $43.6 \%, 47.2 \%, 70.3 \%$ vs $85.4 \%, 89.1 \%, 95.2 \%$; tyrosine-hydroxylase by $30 \%$, $63 \%, 81 \%$ vs $30.7 \%, 70.4 \%, 91.4 \%$; L-aromatic-amino-acid-decarboxylase by $0 \%, 2 \%, 40 \%$ vs $32 \%, 40 \%$, $58 \%$. The prenatal-MPTP may render the DA system sensitive by causing sub-threshold reduction of DA, its metabolites and enzymes, enabling postnatal-MPTP to reduce dopamine above the $70 \%-80 \%$ PD-inducing threshold. Thus, the study may produce a prenatal predisposing/sensitization and postnatal inducing/precipitation model of PD. It also indicates that some cases of PD may have a fetal basis, in which sub-threshold nigrostriatal impairments occur early in life and PD-symptoms are induced during aging by further insults to the dopaminergic system that would not cause PD symptoms in normal individuals.
\end{abstract}

Keywords: Parkinson's Disease; Midbrain; 1-Methyl-4-phenyl-1,2,3,6-tetrahydropyridine (MPTP); Dopamine; Tyrosine Hydroxylase; L-Aromatic Amino Acid Decarboxylase; Sensitization; Precipitation

\section{Introduction}

Parkinson's disease (PD) is an age-associated disorder caused by the death of dopamine (DA) neurons in the substantia-nigra (SN) and DA and tyrosine-hydroxylase depletion in the striatum [1]. The cause for PD is un-known, but the nervous system is a sensitive target for toxic chemicals [2], so interventions that impair/reduce the number of DA cells early-in-life may be a primary underpinning for the occurrence of PD.

Neurons are vulnerable during brain development $[3,4]$ and biological functions decline and cellular death occurs in aging, so, it is hypothesized that idiopathic PD may be initiated during brain development, when toxic factors cause subtle negative changes to the nigrostriatal (NS) DA system, making the affected neuronal pool susceptible/sensitive to harmful interventions encountered later in life. Interestingly, it has been shown that the exposure of pregnant rats to lipopolysaccharide (LPS) reduced DA neurons in the offspring, [5], confirming the proposition that cases of PD may be caused by exposure to toxins [6], including toxins similar to 1-methyl-4-phenyl-1,2,3,6- 
tetrahydropyridine (MPTP) [7,8] and by the interaction of genetic susceptibility with environmental agents $[9,10]$. MPTP is of particular interest since it is the chemical "gold standard" for producing animal models of PD. Its effect in modeling the gastrointestinal dysfunction that occurs frequently in early PD $[11,12]$ further testify to its utility, although the major drawback is that the model syndrome is not of a progressive nature. Pesticide exposure is also a risk-factor for PD [13] and fetal cocaine produced long-term alterations in the dopaminergic system [14]. It is of interest, also, that S-adenosyl-L-methionine (SAM) that is increased during aging caused PD like changes, including tremor, rigidity and hypokinesia when injected into the brain $[15,16]$. SAM is the endogenous methyl donor for DA [17]. And it caused the depletion of striatal DA [18] and the loss of TH and DA cells in the SN [19], so as a metabolite whose activity increases during aging, SAM may contribute to the risk of having PD during aging.

This study is based on the hypothesis that toxin-like substances are involved in the cause of idiopathic PD by inducing, early in life, sub-threshold impairments, that make the neurons unable to withstand the wear-and-tear of life. So, at least two stages of afflictions are involved, the first is the toxic/harmful exposures that occur early in life and sensitize or reduce the number of DA neurons and the second is the later life exposures that cause additional harms to the nigrostriatal dopamine neurons and precipitate the symptoms of PD.

For this project we produced the first, sensitization or predisposing stage by administering MPTP to pregnant mice during the vulnerable stage of the differentiation and neurogenesis of the nigrostriatal DA neurons of the fetuses. For the second, precipitating or inducing stage, the 12th week offspring were challenged with MPTP to test whether the DA neurons in mice exposed to prenatal-MPTP are more affected by postnatal-MPTP. This 2nd stage may serve as a short-term way of mimicking the stress on the DA neurons that occurs during aging.

We studied the midbrain, since it contains the substantia-nigra, the site-of-location for the DA cell bodies, and because the metabolism and the regulation of DA in the cell bodies are different from the striatal axonal terminal field.

\section{Materials and Methods}

\subsection{Animals}

C57BL/6J timed pregnant mice weighing around $20 \mathrm{~g}$ were purchased from Jackson Laboratory, Bar Harbor, Maine, USA. The animal-use was approved by the Institutional Animal Care and Use Committee (IACUC) at Meharry Medical College. The mice were housed 3 per cage under a $12 \mathrm{~h}$ light $12 \mathrm{~h}$ dark cycle, with the light cy- cle occurring from 6 AM to 6 PM. The room is temperature-controlled with standard food and water, ad libitum. The mice were allowed to acclimatize for 6 days before use. The dams were divided into two groups, phosphate buffered saline (PBS) treated group and MPTP treated group. MPTP was purchased from Sigma-Aldrich, St Louis, MO. MPTP was dissolved in PBS and administered intraperitoneally (i.p.). PBS was given at $1.0 \mathrm{ml} / 100 \mathrm{~g}$ body weight and MPTP at $10 \mathrm{mg} / \mathrm{kg}$ at gestation day 8 12 (G8 - G12). MPTP was used as a toxin that targets and harms DA neurons, and the low dose was chosen to cause non-apparent harm to the dams and no physical impairment to the offspring. After delivery the pups were cross-nurtured, and their weights determined, as a way of accessing the global toxic effects of MPTP. At 28 days the pups were weaned and separated according to sex and their weights recorded until the 12th week (Muthian et al., 2010). At 12 weeks the young adult pups from both the prenatal PBS and MPTP groups were randomly divided into four groups each and were treated, intraperitoneally (i.p.) with either PBS, or MPTP $(10,20$ or 30 $\mathrm{mg} / \mathrm{kg}$ ) for 7 days. Seven days after the last MPTP injection the motor activity of the mice was determined and then a sub-group was sacrificed by decapitation, their brains dissected and the midbrain used for Western blot analysis for tyrosine hydroxylase (TH) and L-aromaticamino acid decarboxylase (LAAD) and for the HPLC determination of dopamine and its metabolites, 3,4-dihydroxyphenylacetic acid (DOPAC), 3-methoxytyramine (3-MT) and homovanillic acid (HVA).

\subsection{Measurement of Locomotor Activities}

Seven days after the last postnatal injection of PBS or MPTP the mice were placed in the locomotor activity monitor and measurement started 3 min later and continued for $30 \mathrm{~min}$. The changes in motor activity of the animals were measured in an activity monitor (Versamax Analyzer, Accusccan Instruments, Inc., Columbus, OH, USA). The measurements were performed in a quiet isolated room with dim light. Vertical activity and stereotypy counts were measured and used to represent the natural propensity to move.

\subsection{Western Blot}

Western blot analysis of TH and LAAD protein expression. Immunoblotting was used to quantify the amount of TH and LAAD proteins in the midbrain. After decapitation, the brains were dissected and the sections were homogenized in lysis buffer (Ambion, Austin, TX, USA). Protein concentration was determined using Bio-Rad protein reagent (Bio-Rad, USA). The $20 \mu \mathrm{g}$ proteins were precipitated by adding $100 \%$ methanol and centrifuged at $10,000 \mathrm{rpm}$ for 10 minutes in a Sorvall refrigerated cen- 
trifuge. Then the supernatants were decanted and the precipitates washed with $90 \%$ methanol for 10 minutes. The preparations were subjected to polyacrylamide gel electrophoresis (SDS-PAGE) on a $7.5 \%$ gel. Proteins were dissolved in Laemmli sample buffer and transferred to nitrocellulose membrane. The membranes were blocked in 5\% BSA for one hour. Membranes were incubated with primary rabbit TH or LAAD antibody $(1: 2000$, Chemicon International, CA, USA) and then exposed to the secondary antibody 1:10,000 (HRP conjugated anti-rabbit IgG, Sigma Chemical Co, Saint Louis, MO) and visualized by chemiluminescence. The blots were stripped and reprobed with beta actin antibody to ensure equal protein loading. All the experiments were repeated three times in different animals from the same group to confirm the results.

\subsection{Immunohistochemistry of Tyrosine Hydroxylase}

The mice were anesthetized with chloral hydrate and perfused with cold PBS followed by 4\% paraformaldehyde (PF), prepared in PBS. The brains were postfix for one hour in $4 \% \mathrm{PF}$, removed and placed in cold $15 \%$ sucrose, prepared in PBS, and kept at $4{ }^{\circ} \mathrm{C}$ for $24 \mathrm{hr}$, when they sink. The brains were then frozen in powdered dry-ice and stored at $-70^{\circ} \mathrm{C}$. Thirty microns thick sections were prepared in a cryostat and mounted on gelatin chromealum coated slides. A modified immunohistochemical procedure was used to determine the tyrosine hydroxylase (TH) immunoreactivity (IR). The slide-mounted slices were preincubated in $0.3 \%$ Triton $\mathrm{X}-100$ in PBS, $\mathrm{pH} 7.4$ for three 5-min periods, then incubated in a similar buffer containing rabbit anti-TH serum, approximately 1:1000 dilution and obtained from Chemicon or with non-immune rabbit serum, as control, for about $24 \mathrm{hr}$ at $4^{\circ} \mathrm{C}$. The slides were washed in $0.2 \%$ Triton X-100-PBS buffer for three 5-min periods and incubated in the buffer containing rhodamine-labeled goat antirabbit serum, 1:300 dilution, in reduced light for I hr. The slides were washed in Triton X-100 buffer for one $5 \mathrm{~min}$ period and in the PBS for two $5 \mathrm{~min}$ periods. The sections were drained and coversliped using Fluormont, then viewed and photographed with an epifluorescent equipped microscope.

\subsection{HPLC-Electrochemical (EC) Determination of Dopamine and Its Metabolites}

The isolated midbrain tissues were homogenized in 750 $\mu \mathrm{l}$ of $0.1 \mathrm{M}$ trichloro-acetic acid (TCA), which contains $10^{-2} \mathrm{M}$ sodium acetate, $10^{-4} \mathrm{M}$ EDTA and $10.5 \%$ methanol ( $\mathrm{pH} 3.8)$ using a tissue dismembrator (Fisher Scientific). Samples were spun in a micro centrifuge at 10,000 $\times \mathrm{g}$ for 20 minutes. Samples of the supernatant were then analyzed for dopamine and its metabolites at the core analytical facility at Vanderbilt University. Dopamine and its metabolites were determined by specific HPLC assay utilizing an Antec Decade 11 (oxidation: 0.5) electrochemical detector operated at $33^{\circ} \mathrm{C}$. Supernatant samples of $20 \mu \mathrm{l}$ were injected, using a Water's 717 + autosampler, onto a Phenomenex Nucleosol (5 U, 100 A) C18 HPLC column $(150 \times 4.60 \mathrm{~mm})$. Biogenic amines were eluted with a mobile phase consisting of $89.5 \% 0.1 \mathrm{M}$ TCA, $10^{-2} \mathrm{M}$ sodium acetate, $10^{-4} \mathrm{M}$ EDTA and $10.5 \%$ methanol ( $\mathrm{pH} \mathrm{3.8).} \mathrm{Solvent} \mathrm{is} \mathrm{delivered} \mathrm{at} 0.8 \mathrm{ml} / \mathrm{min}$ using Water's 515 HPLC pump. Using this HPLC solvent the following biogenic amines elute in the following order: noradrenaline, adrenaline, di-hydroxyphenylacetic acid (DOPAC), dopamine (DA), 5-hydroxyindole acetic acid (5-HIAA), 5-hydroxytryptamine (5-HT), and 3-methoxytyramine (3-MT). HPLC control and data acquisition were managed by Water's Empower software.

\subsection{Statistical Analysis}

All data are reported as mean \pm SEM and analyzed by one way analysis of variance (ANOVA) followed by Newman-Keuls test. P values less than 0.05 were considered significant. (PRISM, Graphpad Software Inc.).

\section{Results}

\subsection{Effects of Prenatal MPTP on the Activity of C57BL/6J Mice Offspring}

The vertical and stereotype activities were used to determine the general movement propensity of the prenatalPBS and prenatal-MPTP exposed offspring. The prenatal-MPTP exposed offspring showed significant reducetion in vertical activity (Figure 1(a)) and stereotypy counts (Figure 1(b)) when compared to prenatal-PBS exposed offspring.

\subsection{Prenatal MPTP-Exposure on Midbrain DA, DOPAC, HVA, 3-MT and TH and LAAD}

The concentrations of dopamine (DA), 3,4-dihydroxyphenylacetic acid (DOPAC), 3-methoxytyramine (3-MT) and homovanillic acid (HVA) were measured in the midbrain of 12 weeks old $\mathrm{C} 57 \mathrm{BL} / 6 \mathrm{~J}$ offspring that were exposed in utero at G8-12 to $10 \mathrm{mg} / \mathrm{kg}$ MPTP or to PBS. The prenatal treatments with MPTP reduced the levels of DA by $49.7 \%(16.5 \pm 3.5$ to $8.3 \pm 2.9 \mathrm{ng} / \mathrm{mg}$ protein $)$, DOPAC by $48 \%,(2.5 \pm 0.35$ to $1.3 \pm 0.25 \mathrm{ng} / \mathrm{kg}$ protein $)$ and 3 -MT by $52.2 \%,(2.3 \pm 0.12$ to $1.1 \pm 0.10 \mathrm{ng} / \mathrm{kg}$ protein). HVA level was statistically unchanged, showing a slight increase of $11 \%$ (Table 1). The table also shows that in utero exposure to MPTP reduced the protein density for $\mathrm{TH}$ by $20.9 \%$ (also Figure 3 bands 2 ) and for LAAD by $25 \%$ (also Figure 4, bands 2), as determined by Western blot detection technique. 


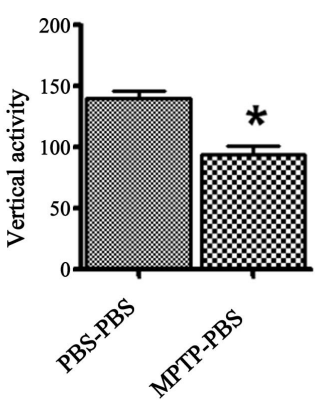

(a)

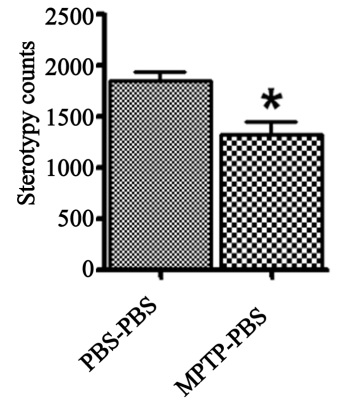

(b)
Figure 1. The effect of prenatal MPTP on motor activities in C57BL/6J mice. The measurements were made 12 weeks after MPTP exposure in prenatal MPTP or PBS exposed offspring. The vertical activity and sterotypy counts were measured using an activity monitor (Versamax Analyzer, Accuscan Instruments Inc., Columbus, OH, USA) Values were expressed as mean \pm SEM of 6 animals. Data were analysed by student $t$ test with $P<0.05$, and with asterisk (*) indicating significant differences of the prenatal-MPTP group from the prenatal-PBS group.

\subsection{The Effects of Postnatal MPTP Challenges on DA, DOPAC, 3-MT and HVA in Mice Offspring Exposed to Prenatal MPTP or to Prenatal PBS}

MPTP was administered to 12 weeks old mice offspring that were exposed in utero to MPTP or to PBS, in order to determine if the exposure to MPTP, used as a model toxin for nigrostriatal dopamine neurons, causes additional or potentiating toxic effects later in the life of the prenatal-MPTP exposed offspring. Table 2 shows that the postnatal MPTP reduced DA in a dose-dependent fashion in both the in utero or prenatal exposed PBS control and the in utero exposed MPTP offspring. For the prenatal-PBS exposed control groups, the doses of 10, 20 and $30 \mathrm{mg} / \mathrm{kg}$ of postnatal-MPTP reduced midbrain DA from a control value of $16.5 \pm 3.5 \mathrm{ng} / \mathrm{mg}$ protein to $9.3 \pm$ $2.0 \mathrm{ng} / \mathrm{kg}, 8.7 \pm 1.8 \mathrm{ng} / \mathrm{kg}$ and $4.9 \pm 0.8 \mathrm{ng} / \mathrm{mg}$ protein, respectively (Table 2). The same doses of postnatalMPTP administered to mice exposed to $10 \mathrm{mg} / \mathrm{kg}$ of prenatal-MPTP reduced DA from the prenatal-MPTPpostnatal-PBS level of $8.3 \pm 2.9 \mathrm{ng} / \mathrm{mg}$ protein to $2.4 \pm$ $2.9 \mathrm{ng} / \mathrm{mg}, 1.8 \pm 0.9 \mathrm{ng} / \mathrm{mg}$ and $0.8 \pm 0.2 \mathrm{ng} / \mathrm{mg}$ protein, respectively (Table 2). The values for the prenatal-PBS exposed group represent dose-related reductions of $43.6 \%, 47.3 \%$ and $70.3 \%$ versus the reductions of the values for the prenatal-MPTP exposed offspring of $71.1 \%, 78.3 \%$ and $90.4 \%$. The percent depletion of DA in PD cases is reported against the normal or population striatal DA level, therefore, when the reduction caused by postnatal-MPTP is compared to the value of 16.5 $\mathrm{ng} / \mathrm{mg}$ for the prenatal-PBS-postnatal-PBS control or for the normal population, the depletion of DA caused by the
10,20 and $30 \mathrm{mg} / \mathrm{kg}$ of postnatal-MPTP represents 43.6, 47.3 and 70.3 for the prenatal-PBS offspring that received postnatal-MPTP versus $85.4 \%, 89.1 \%$ and $95.2 \%$ for the prenatal-MPTP offspring that received postnatal-MPTP treatments (Table 2). The data, therefore, show that the differences in the reduction of DA caused by the 10,20 and $30 \mathrm{mg} / \mathrm{kg}$ of postnatal-MPTP were $41.8 \%$, $41.9 \%$ and $24.9 \%$ for the offspring exposed to prenatal-PBS versus those exposed to prenatal-MPTP. Such an outcome shows that postnatal-MPTP has a greater effect in reducing DA levels in the offspring exposed to prenatal-MPTP, as compared to the offspring exposed to prenatal-PBS and indicating that the prenatal-MPTP exposure sensitizes the nigrostriatal dopamine system of the offspring.

Postnatal-MPTP caused dose-related reduction in the levels of DOPAC and 3-MT in both the prenatal-PBS and prenatal-MPTP groups. Similar to the effect on DA, postnatal-MPTP treatment also caused more marked reduction of DOPAC and 3-MT in the mice exposed to prenatal-MPTP, as compared to the group exposed to prenatal-PBS (Table 2). For DOPAC, the differences between prenatal-PBS and prenatal-MPTP are $40.8 \%$, $42.8 \%$ and $20.0 \%$ caused by the 10,20 and $30 \mathrm{mg} / \mathrm{kg}$ postnatal MPTP. For 3-MT, the differences were $45.6 \%$, $30.9 \%$ and $26.6 \%$ for the prenatal-PBS vs prenatalMPTP offspring treated with 10,20 and $30 \mathrm{mg} / \mathrm{kg}$ of postnatal-MPTP. The changes in HVA did not show a dose-effect relationship nor differences between the prenatal-PBS and prenatal-MPTP exposed animals. The levels of DA, DOPAC, 3-MT and HVA are illustrated in Figures 2(a)-(d) and highlight the dose-related reduction of DA, DOPAC and 3-MT following postnatal-MPTP. The figure also illustrates the more severe reductions in the mice that were exposed to prenatal-MPTP (striped columns) as compared to mice exposed to prenatal-PBS (filled columns). Postnatal MPTP reduced HVA in both prenatal treated groups, but a dose-effect relationship did not occur (Figure 2(d)).

\subsection{Analyzing the Effects of Postnatal-MPTP by Normalizing (percentage) the Changes Seen in the Prenatal-MPTP and Prenatal-PBS Mice}

In order to evaluate and compare the relative changes caused by the prenatal-MPTP exposure, as compared to the prenatal-PBS exposure, we normalized the values for DA, DOPAC, HVA and 3-MT based on assigning 100\% as the base value for the postnatal-PBS control (0 MPTP) for both the prenatal-PBS and prenatal-MPTP exposed groups. The percent values derived for the 10,20 and 30 $\mathrm{mg} / \mathrm{kg}$ postnatal-MPTP for both the prenatal-PBS and prenatal-MPTP animals are plotted and are shown in 
Table 1. Midbrain DA, DOPAC, 3-MT, HVA, TH and LAAD in offspring exposed in utero to MPTP ${ }^{\dagger}$.

\begin{tabular}{|c|c|c|c|c|c|c|}
\hline \multicolumn{7}{|c|}{ Dopamine and metabolite (ng/mg protein) } \\
\hline Treatment group & DA & DOPAC & $3 \mathrm{MT}$ & HVA & $\mathrm{TH}$ & LAAD \\
\hline МРТР, $10 \mathrm{mg} / \mathrm{kg}$ & $8.3 \pm 2.9^{*}$ & $1.3 \pm 0.25^{*}$ & $1.1 \pm 0.10^{*}$ & $4.0 \pm 0.40$ & $79.1 \%$ & $75 \%$ \\
\hline
\end{tabular}

${ }^{\dagger}$ The pregnant dams were injected with $10 \mathrm{mg} / \mathrm{kg}$ MPTP or with PBS during gestation days 8 - 12 to expose the brain of the fetus to MPTP. The offspring were sacrificed at 3 months of age and midbrain DA, DOPAC, HVA and 3-MT were determined by HPLC and tyrosine hydroxylase (TH) and L-aromatic amino acid decarboxylase (LAAD) detected and quantified via Western blot; ${ }^{*}$ indicate the significant changes at $\mathrm{P} \leq 0.05$, caused by the prenatal-MPTP exposure as compared to prenatal-PBS exposured offspring. The values are expressed \pm SEM of 6 animals for dopamine and it metabolites. TH and LAAD values are expressed as mean \pm SEM for 3 animals. Data were analyzed by one way analysis of variance followed by Newman-Keuls test.

Table 2. The effects of postnatal-MPTP of 10,20 and $30 \mathrm{mg} / \mathrm{kg}$ or PBS on midbrain DA, DOPAC, 3-MT and HVA in offspring exposed to prenatal-MPTP or prenatal-PBS.

\begin{tabular}{cccccc}
\hline $\begin{array}{c}\text { DA and metablites } \\
(\mathrm{ng} / \mathrm{mg} \text { protein) }\end{array}$ & Prenatal exposure & & \multicolumn{2}{c}{ Postnatal PBS or MPTP challenges (mg/kg) } \\
\hline & & PBS & $10 \mathrm{mg} / \mathrm{kg}$ & $20 \mathrm{mg} / \mathrm{kg}$ & $30 \mathrm{mg} / \mathrm{kg}$ \\
DA & PBS & $16.5 \pm 3.5[0.0]$ & $9.3 \pm 2[43.6]^{*}$ & $8.7 \pm 1.8[47.2]^{*}$ & $4.9 \pm 0.8[70.3]^{*}$ \\
& MPTP, $10 \mathrm{mg} / \mathrm{kg}$ & $8.3 \pm 2.9[49.7]$ & $2.4 \pm 0.4[85.4]^{*}$ & $1.8 \pm 0.9[89.1]^{*}$ & $0.8 \pm 0.2[95.2]^{*}$ \\
DOPAC & PBS & $2.5 \pm 0.35[0.0]$ & $1.9 \pm 0.3[+24]$ & $1.5 \pm 0.2[40.0]^{*}$ & $0.58 \pm 0.08[76.8]^{*}$ \\
& MPTP, $10 \mathrm{mg} / \mathrm{kg}$ & $1.3 \pm 0.25[48]$ & $0.88 \pm 0.2[64.8]^{*}$ & $0.43 \pm 0.1[82.8]^{*}$ & $0.08 \pm 0.07[96.8]^{*}$ \\
$3-\mathrm{MT}$ & PBS & $2.3 \pm 0.12[0.0]$ & $1.9 \pm 0.2[17.4]$ & $1.3 \pm 0.2[43.4]^{*}$ & $0.61 \pm 0.1[73.4]^{*}$ \\
& MPTP, $10 \mathrm{mg} / \mathrm{kg}$ & $1.1 \pm 0.10[52.2]$ & $0.85 \pm 0.1[63]^{*}$ & $0.59 \pm 0.08[74.3]^{*}$ & $0.00 \pm 0.0[100]^{*}$ \\
HVA & PBS & $3.6 \pm 0.20[0.0]$ & $2.4 \pm 0.2[33.3]$ & $2.5 \pm 0.2[30.5]$ & $2.2 \pm 0.1[38.8]$ \\
& MPTP, $10 \mathrm{mg} / \mathrm{kg}$ & $4.0 \pm 0.40[0.0]$ & $2.3 \pm 0.3[36.1]$ & $2.5 \pm 0.3[30.5]$ & $2.5 \pm 0.4[36.6]$ \\
\hline
\end{tabular}

The percent changes, based on the normal population (prenatal-PBS plus postnatal-PBS) levels are enclosed in brackets. The results show that postnatal-MPTP caused dose-related reduction in DA, DOPAC and 3-MT in both groups, but postnatal MPTP shows more toxic effects in reducing DA, DOPAC and 3-MT in the prenatal-MPTP offspring as compared to the prenatal-PBS offspring. The outcome, therefore, suggests that the prenatal-MPTP exposure sensitizes the midbrain to the toxic effects of postnatal MPTP. Data were analyzed by using one-way analysis of variance followed by Newman-Keuls test. Asterisks $\left(^{*}\right)$ indicate the significant dose-related effect of postnatal-MPTP for both prenatal-PBS and prenatal-MPTP exposed groups.

Figure 3. The figure shows that DA was depleted to a greater extent in the prenatal-MPTP exposed mice as compared to the prenatal-PBS offspring: noting that the dose-effect plot for the prenatal-MPTP offspring falling below the plot for the prenatal-PBS offspring (Figure 3(a)). Thus, the plot shows that the exposure to prenatalMPTP, as compared to prenatal-PBS, renders the midbrain dopaminergic system susceptible to the effects of postnatal-MPTP. Similar prenatal-MPTP sensitization is shown for DOPAC, at the 20 and $30 \mathrm{mg} / \mathrm{kg}$ dose levels of postnatal-MPTP (Figure 3(b)) and only at the 30 $\mathrm{mg} / \mathrm{kg}$ of postnatal-MPTP for 3-MT (Figure 3(c)). So, DA showed sensitization to prenatal-MPTP exposure at all dose levels, but the metabolites, DOPAC and 3-MT were sensitive only at the higher dosage of post-natal MPTP.

\subsection{Postnatal-MPTP Effects on Midbrain TH Expression in Prenatal-PBS and Prenatal-MPTP Exposed Offspring}

Figure 4 shows the effects of postnatal-MPTP administration on the expression of $\mathrm{TH}$ in the midbrain of the offspring of C57BL/6J mice exposed to prenatal-PBS or to prenatal-MPTP during gestation day 8 - 12 (G8 - G12). The figure shows that prenatal-MPTP reduced midbrain TH by $20.9 \%$ (Figure 4(a), blots 1 vs 2 , and 4(b), 1 st vs 2nd columns). Shown also in Figure 3 are the effects of $0,10,20$ and $30 \mathrm{mg} / \mathrm{kg}$ of postnatal-MPTP administered to the 12 weeks old offspring that were exposed to prenatal-PBS (Figure 4(a), blots 1, 3, 5, 7 and 4(b), filled columns) or to prenatal-MPTP (Figure 4(a), blots 2, 4, 6, 8 and 4(b), striped columns). The postnatal-MPTP caused dose-dependent reduction of TH in both groups. Doses of 


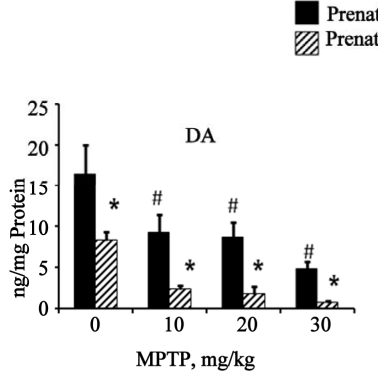

(a)

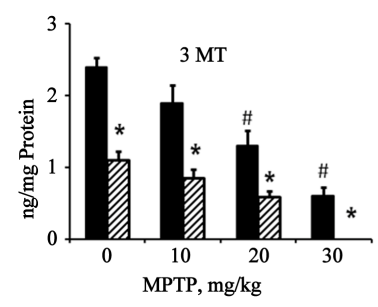

(c)

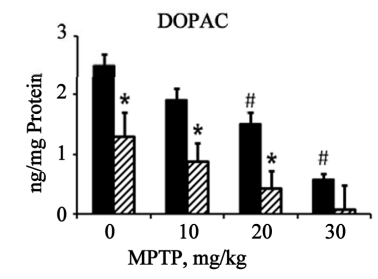

(b)

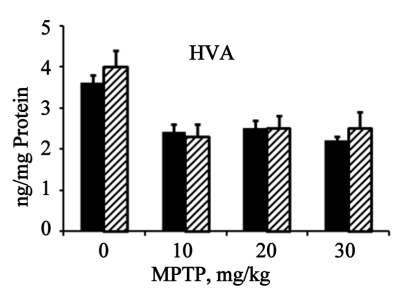

(d)
Figure 2. The effects of postnatal doses of 0 (PBS), 10, 20 and $30 \mathrm{mg} / \mathrm{kg}$ of MPTP on midbrain DA, DOPAC, HVA and 3-MT in C57BL/6J mice offspring that were exposed to prenatal-PBS or prenatal-MPTP. Figure 2(a)-(d) represent the changes in DA, DOPAC, 3-MT and HVA. PostnatalMPTP causes dose-dependent reduction in DA, DOPAC and 3-MT in both groups (Figures 2(a)-(c)), however, postnatal-MPTP showed greater effects in the reduction DA, DOPAC and 3-MT levels in mice exposed to prenatalMPTP (striped columns) as compared to prenatal-PBS (filled columns). Postnatal-MPTP reduced HVA but not in a dose-related fashion (Figure 2(d)). Asterisks (*) indicates significant difference, at $P \leq 0.05$, caused by postnatalMPTP in the prenatal-PBS offspring as compared to the prenatal-MPTP group, and pounds (\#), highlight the significant reduction caused by the postnatal MPTP treatment. The values are expressed in $\mathrm{ng} / \mathrm{mg}$ protein and as mean \pm SEM for 6 animals. Data were analyzed by using one-way analysis of variance followed by Newman-Keuls test. GraphPad Prism 5 software was used in the analysis.

10,20 and $30 \mathrm{mg} / \mathrm{kg}$ of MPTP reduced mid- brain TH by $30 \%, 63 \%$ and $81 \%$, respectively, in the prenatal-PBS control (Figure 4, blots, 3, 5, 7 and 4(b), 2nd, 3rd, 4th filled columns). In the offspring exposed to prenatalMPTP the same MPTP doses reduced midbrain TH by $30.7 \%, 70.4 \%$ and $91.4 \%$, respectively, (Figure 4, blots 4, 6, 8 and 4(b), 2nd, 3rd, 4th striped columns). When the data were normalized the curves statistically overlapped (Figure 4(c)), which means that the midbrain $\mathrm{TH}$ did not show sensitivity to the postnatal-MPTP challenges.

\subsection{Substantia Nigra TH Immunoreactivity in Prenatal-MPTP as Compared to Prenatal-PBS Exposed Offspring}

Figure 5 shows the effect of prenatal MPTP exposure on

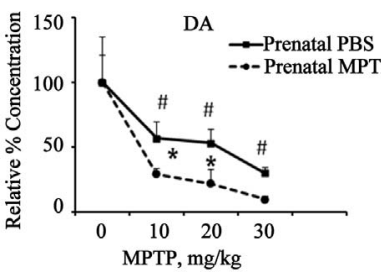

(a)

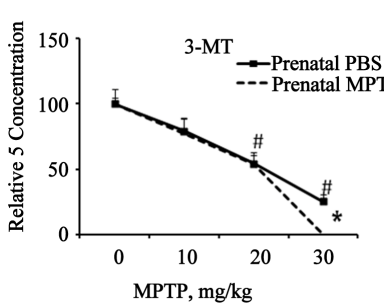

(c)

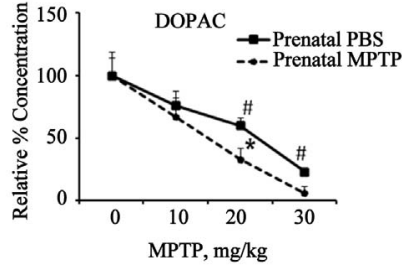

(b)

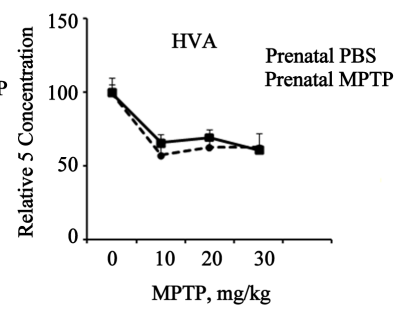

(d)
Figure 3. Normalized plots are shown to highlight the relative effects of postnatal-MPTP on DA, DOPAC, 3-MT and HVA in mice exposed to prenatal-PBS or prenatal-MPTP. The plots show the percent concentrations plotted against the $0,10,20$ and $30 \mathrm{mg} / \mathrm{kg}$ of postnatal-MPTP for the prenatal-PBS and prenatal-MPTP groups. The significant separation of the curves with the curve for the prenatalMPTP groups laying below the curve for the prenatal-PBS offspring indicates that prenatal-MPTP causes sensitization to the postnatal-MPTP treatment. The vertical separation of the curves is indicative of the degree of sensitization. The data show that marked sensitization occurs for DA at all postnatal-MPTP dose levels but only at the $30 \mathrm{mg} / \mathrm{kg}$ for 3-MT. Asterisks (*) indicates significant changes at $P \leq 0.05$ caused by postnatal-MPTP in the prenatal-PBS versus the prenatal-MPTP offspring and pounds (\#) the significant dose-effect of postnatal-MPTP for both groups. The values are expressed in $\mathrm{ng} / \mathrm{mg}$ protein and as mean \pm SEM of 6 animals. Data were analyzed by using one way analysis of variance followed by Newman-Keuls Test. GraphPad Prism 5 software was used in the analysis.

TH immunoreactivity in the substantia nigra of the midbrain of $\mathrm{C} 57 \mathrm{BL} / 6 \mathrm{~J}$ mice. The offspring exposed to prenatal-MPTP during gestation day 8 - 12 (G8 - G12) showed a reduction in $\mathrm{TH}$ positive cells in the substantia nigra (Figure 5, left), when compared to prenatal-PBS treated groups (Figure 5, right).

\subsection{Postnatal-MPTP Effects on Midbrain LAAD Expression in Prenatal-PBS and Prenatal-MPTP Exposed Offspring}

Figure 6 shows the effects of $0,10,20$ and $30 \mathrm{mg} / \mathrm{kg}$ of postnatal-MPTP on LAAD expression in the midbrain of C57BL/6J mice offspring exposed to prenatal-PBS (Figure 6(a), blots 1, 3, 5, 7 and 6(b), 1st, 2nd, 3rd, 4th filled columns) or to prenatal-MPTP (Figure 6(a), blots 2, 4, 6, 8 and $\mathbf{6 ( b )}, 1$ st, 2nd, 3rd, 4th striped columns). In the offspring that were exposed to prenatal-PBS the 10 and 20 


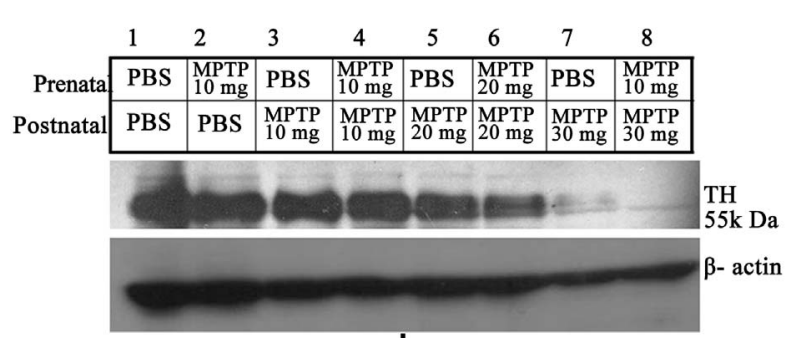

(a)

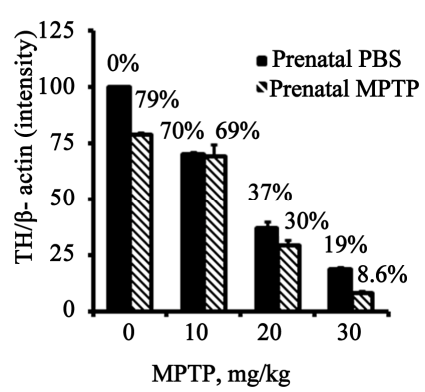

(b)

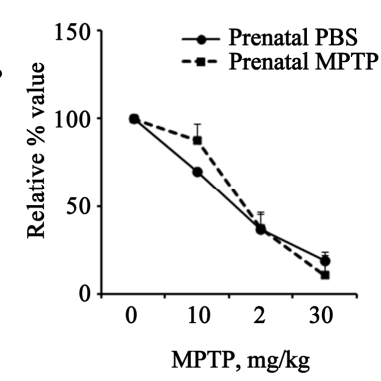

(c)
Figure 4. Immunoblot and densitometric analysis of midbrain TH for mice exposed to prenatal-PBS or prenatalMPTP and challenged at 3 months with doses of $0,10,20$ or $30 \mathrm{mg} / \mathrm{kg}$ MPTP. The prenatal-PBS groups are represented in bands 1, 3, 5 and 7 and the filled columns in Figure 4. The $10 \mathrm{mg} / \mathrm{kg}$ prenatal-MPTP groups are represented by bands 2, 4, 6, and 8 and stripe columns in Figure 4(b). Postnatal-MPTP caused dose-related reduction of TH in both prenatal-PBS and prenatal-MPTP offspring (Figure 4(a) and (b)). The plot of the relative/normalized outcome is presented in Figure 4(c) and shows that the curves overlap, indicating that the prenatal exposure to MPTP did not sensitize midbrain TH to postnatal-MPTP. The values are expressed as mean \pm SEM of 3 animals.
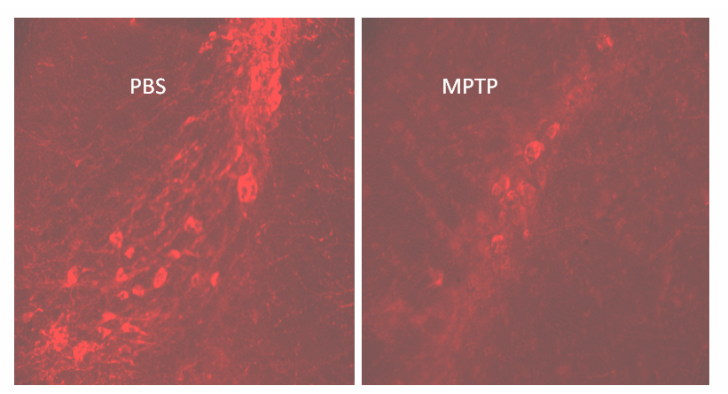

Figure 5. Image showing tyrosine hydroxylase immunoreactivity (TH-1) in the rostral portion of the substantia nigra of the 12 weeks old offspring exposed to prenatal PBS (left) or MPTP (right).

$\mathrm{mg} / \mathrm{kg}$ dose levels of MPTP showed no appreciable effect, but the $30 \mathrm{mg} / \mathrm{kg}$ dose level reduced midbrain LAAD by $40 \%$ (Figure 6(a), blot 7 and 6(b), 4 th filled column). In the offspring that were exposed to prenatalMPTP the reductions were 32, 40 and 58\% (Figure 6(a), blots 4, 6, 8 and B, 2nd, 3rd, 4th striped columns) for the 10,20 and $30 \mathrm{mg} / \mathrm{kg}$ of MPTP. So, as compared to the
prenatal-PBS groups, the LAAD of the mice exposed to prenatal-MPTP was more affected by the postnatal-PTP. The normalized curves that define the relative effects of postnatal-MPTP for both groups indicate that the prenatal-MPTP groups were slightly, but not statistically more sensitive to the postnatal MPTP (Figure 6(c)), as indicated by the slight difference seen with the 10 and 20 $\mathrm{mg} / \mathrm{kg}$ dose level. The $30 \mathrm{mg} / \mathrm{kg}$ dose was appreciably toxic to the prenatal-PBS group, as well (Figure 6(c)).

\section{Discussion}

The midbrain contains the substantia nigra that is composed mostly of dopamine neuronal cell bodies that project to the neostriatum. The degeneration of these neurons, resulting in about $70 \%-80 \%$ loss of DA in the striatum causes the symptoms of PD. Although the DA neuronal cell bodies and their projecting fibers and terminals form one system, the proportion of the enzymes and metabolites contained in both the midbrain and the

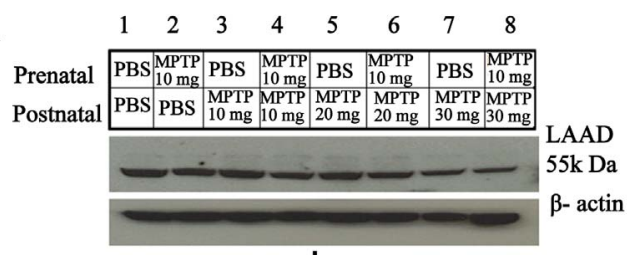

(a)

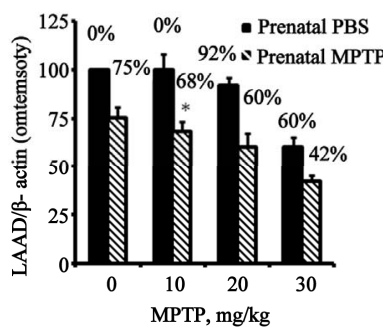

(b)

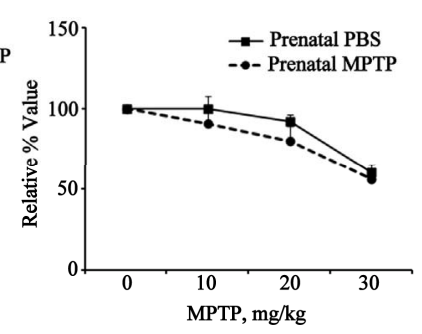

(c)
Figure 6. Immunoblot and densitometric analysis of midbrain LAAD for mice exposed to prenatal-PBS or prenatal-MPTP and challenged at 3 months with doses of 0,10 , 20 or $30 \mathrm{mg} / \mathrm{kg}$ MPTP. The prenatal-PBS groups are represented in bands $1,3,5$ and 7 and the filled columns in $B$ in figure 6. The $10 \mathrm{mg} / \mathrm{kg}$ prenatal-MPTP groups are represented by bands $2,4,6$, and 8 and striped columns in figure 6. Postnatal-MPTP caused dose-related reduction of LAAD in both prenatal-PBS and prenatal-MPTP offspring (figures 6(a) and (b)). The plot of the relative, normalized or percent outcome in Figure 6(c), shows that the curves for the prenatal-MPTP offspring falls below the curve for the prenatal-PBS exposed offspring, indicating that midbrain TH for the offspring exposed to prenatal-MPTP was more slightly affected by the postnatal-MPTP at the 10 and $20 \mathrm{mg} / \mathrm{kg}$ dose levels. The steep deflection means that the $30 \mathrm{mg} / \mathrm{kg}$ dose-level was equally toxic to the prenatal-PBS offspring as well. The values are expressed as mean \pm SEM of 3 animals. 
striatal regions are quite different. This is apparently due to functional differences and specialized processes, such as protein synthesis in the cell body, anterograde transport of enzymes to the nerve terminal and retrograde transport of DA to the cell body. This study found that the concentration of DA in the midbrain is $16.5 \pm 3.5$ $\mathrm{ng} / \mathrm{mg}$ protein, which is 9.53 times less than the concentration of $157.3 \pm 17.3 \mathrm{ng} / \mathrm{mg}$ protein, measured in the striatum [20]. The analysis of the midbrain also showed that DA was reduced by $49.7 \%$ or by $8.2 \mathrm{ng} / \mathrm{mg}$ protein following the prenatal-MPTP, whereas in the striatum the reduction by the same exposure was a non-significant $13.80 \%$ [20]. This $13.80 \%$ however, represents a DA concentration change of $21.7 \mathrm{ng} / \mathrm{mg}$ protein. This shows that the greater percent reduction of DA in the midbrain was related and the lower level of DA in the cell body region in the midbrain. The lower level of DA in the midbrain is expected since DA is synthesized in nerve endings in the striatum. It is unknown, though, whether DA has a major function or regulatory role in the cell body of DA neurons in the midbrain.

The outcome also reveals that DA represents $66.26 \%$ and the metabolites $33.74 \%$ of the midbrain total amounts. For the striatum, DA was shown to represent $86.28 \%$ and the metabolites were $13.71 \%$ of the midbrain total amount of DA and its metabolites. So, the biodisposition of DA and its metabolites is different in the midbrain and the striatum, and they may be affected differently by external interventions, but the prenatal-MPTP exposure markedly affects the methylation of DA in both regions, as shown by $52.2 \%$ reduction of 3 -MT in the midbrain and a $66.25 \%$ reduction was observed in the striatum [20].

In the study MPTP was used as the prenatal toxin, since it is known to damage DA neurons. A low dose of MPTP was used to target the emerging and developing dopamine neurons in the fetuses. The purpose was to determine whether moderate and early impairments to the DA neurons would modify those cells so that they become vulnerable to insults that they encounter later in life, thus producing a model for PD that may also hint to the etiology of some forms of human PD. The study, therefore, test a hypothesis that two stages are involved in the etiology of some forms of PD: a predisposing or sensitization stage that occurs early in life, causing subtle harm to the nigrostriatal dopaminergic system so that they become susceptible or vulnerable, and the second stage, which is the precipitating or inducing stage, occurs later in life when additional insults to the neurons cause further or additional harm to the nigrostriatal dopaminergic system.

The results obtained support the hypothesis, because the prenatal-MPTP exposure caused changes including the sub-threshold reduction of DA, in which the offspring appear normal in spite of the $49.7 \%$ reduction in midbrain DA and $20.9 \%$ reduction in TH (Table 1). When the adult mice offspring were challenged with MPTP at 3 months to mimic the precipitating stage, changes in DA and its metabolites were markedly and more severely affected in the offspring exposed to prenatal-MPTP, as compared to the offspring exposed to prenatal-PBS. Using the $70 \%$ reduction of DA as the threshold at which PD symptoms occurs, it is clear that all postnatal doses of MPTP administered to the offspring that were exposed to prenatal-MPTP caused parkinsonism-inducing reduction of DA, in the range of $85 \%-95 \%$, whereas, only the highest dose level, of $30 \mathrm{mg} / \mathrm{kg}$ of MPTP, administered to the prenatal-PBS offspring caused DA reduction of $70 \%$. When the data are normalized and plotted to show relative effects in the prenatal-PBS versus prenatalMPTP groups, the plot of the changes in DA (Figure 3(a)) indicates that the prenatal-MPTP group was more sensitive to postnatal-MPTP. Similar plots for TH (Figure 4(c)) and LAAD (Figure 5(c)) suggest that the change in DA is not related to $\mathrm{TH}$, and apparent decline in tyrosine hydroxylase immunoreactivity in prenatal MPTP treated offspring but is more aligned to the pattern of change shown for LAAD.

This study, therefore, supports the hypothesis that a predisposing or sensitization condition may exist for some individuals that develop PD and that postnatal stress imposed on the basal ganglia later in life may cause additive effects that precipitate the symptoms of PD. The pathology of PD, however, extends beyond the basal ganglia impairments, that are responsible for the movement related symptoms. Gastrointestinal impairment, such as constipation, is now well known to occur in PD and before the onset of the movement disorders. Depression also often accompanies the movement related abnormalities in PD. So, it would be of interest to know, in a PD model, whether the impairments of neurons that control gastrointestinal motility, and brain neurons that control mood, have a prenatal origin. Interestingly, the MPTP toxin that we used in the model was effective in causing PD-like changes and parallel impairments to gastrointestinal $\mathrm{TH}$-positive neurons [12] and to frontal cortex TH-positive neurons [21] that together may help to explain the co-morbid occurrence of gastrointestinal impairments [12] and depression [21] with PD.

The results also show that prenatal exposure to MPTP, at a concentration that has no visible toxic effect on the dams, has long-lasting effect in the offspring, evidenced by the effects on DA, TH and LAAD. This indicates that other toxins, excesses of otherwise safe chemicals and nutritional deficiency may play a predisposing role in the cause of PD, if the incidences of exposure pair with the vulnerable stage of the $\mathrm{TH}$ regulatory neurons. 


\section{Conclusion}

The objective of this study was to test the hypothesis that two sets or two stages of impairments can produce a PDlike model disorder. The first stage was represented by the prenatal exposure to the DA toxin, MPTP, and the second stage involved challenging the offspring with MPTP during adulthood, and to determine whether the offspring exposed to the prenatal MPTP will show a greater degree of toxicity to postnatal-MPTP challenge. The results show that prenatal-MPTP exposure caused sub-threshold deficiencies in the nigrostriatal dopamine neuronal system, and that the degrees of toxicity caused by the postnatal-MPTP challenges in the prenatal-MPTP exposed offspring were more severe, when compared to the prenatal-PBS offspring. This is a positive test for the hypothesis. What were revealed in this study, also, are the differences in the bio-disposition of dopamine and its metabolites in the midbrain, as compared to the striatum [20], although both structures represent the one nigrostriatal dopamine system. DA level was 9.5 times lower in the midbrain, as compared to the striatum [20], which may explain the fact that the site for the synthesis of DA is in the striatum, and that the retrograde transport of DA is limited. The higher proportion of the total DA metabolites of $33.74 \%$ in the midbrain (Table 1) as compared to $13.71 \%$ in the striatum [20] may also reflect the tendency towards increased DA metabolism in the midbrain DA cell bodies, where DA is not protected by vesicular uptake. The quantitative disparity between the striatal and midbrain DA system means that it is best to treat the midbrain DA compartment differently from the striatal DA compartment. The super-imposition of a slowly developing postnatal stress, such as aging, on the basal ganglia, as compared to the acute toxicity of MPTP, may present a postnatal challenge that is more appropriate for modeling PD.

\section{Acknowledgements}

This work was supported by NIH RO1NS041674, NIH R21NS049623, NIH 5U01NS 041071, NIH

5R25GM059994 and by a gift from Bernard Crowell, MD., PhD., Little Rock, A.R.

\section{REFERENCES}

[1] R. E. Heikkila and P. K. Sonsalla, "The MPTP Treated Mouse as a Model of Parkinsonism: How Good Is It?" Neurochemistry International, Vol. 20, 1992, pp. 299-303. doi:10.1016/0197-0186(92)90256-Q

[2] P. Grandjean and P. J. Landrigan, "Developmental Neurotoxicity of Industrial Chemicals.” Lancet, Vol. 368, No. 9553, 2006, pp. 2167-2178. doi:10.1016/S0140-6736(06)69665-7

[3] H. R. Andersen, J. B. Nielsen and P. Grandjean, "Toxi- cologic Evidence of Developmental Neurotoxicity of Environmental Chemicals," Toxicology, Vol. 144, No. 1-3, 2000, pp. 121-127. doi:10.1016/S0300-483X(99)00198-5

[4] L. G. Costa. M. Aschner, A. Vitalone, T. Syversen and O. P. Soldin, "Developmental Neuropathology of Environmental Agents," Annual Review of Pharmacology and Toxicology, Vol. 44, No. 1, 2004, pp. 87-110. doi:10.1146/annurev.pharmtox.44.101802.121424

[5] Z. Ling, D. A, Gayle, S.Y. Ma, J. W. Lipton, C. W. Tong, J. S. Hong and P. M. Carvey, "In Utero Bacterial Endotoxin Exposure Causes Loss of Tyrosine Hydroxylase Neurons in the Postnatal Rat Midbrain," Movement Disorders, Vol. 17, No. 1, 2002, pp. 116-124.

doi: $10.1002 / \mathrm{mds} .10078$

[6] D. B. Calne and J. W. Langston, "Etiology of Parkinson's Disease," Lancet, Vol. 322, No. 8365, 1983, pp. 1457 1459. doi:10.1016/S0140-6736(83)90802-4

[7] P. H. V. Jenner, A. Shapira and C. D. Marsden, "New Insight in to the Cause of Parkinson's Disease". Neurology, Vol. 42, No. 12, 1992, pp. 2241-2250. doi:10.1212/WNL.42.12.2241

[8] K. Aoyama, M. Matsubara, Y. Kondo, M. Murakawa, K. Suno and S. Yamaguchi, "N-Methylation Ability for Azaheterocyclic Amines in Higher in Parkinson's Disease: Nicotinamide Loading Test," Journal of Neural Transmission, Vol. 107, No. 8-9, 2000, pp. 985-995. doi:10.1007/s007020070047

[9] D. A. Di Monte, “The Environment and Parkinson's Disease: Is the Nigrostriatal System Preferentially Targeted by Neurotoxins," The Lancet Neurology, Vol. 2, No. 9, 2003, pp. 531-538.

[10] J. L. Kennedy, L. A. Farrer, N. C. Andreason, R. Mayeux and P. St. George Hyslop, "The Genetics of Adult-Onset Neuropsychiatric Disease: Complexities and Conundra?" Science, Vol. 302, No. 5646, 2003, pp. 822-826. doi:10.1126/science.1092132

[11] G. Anderson, A. R. Noorian, G. Taylor, M. Anitha, D. Bernhrd, S. Srinivasan and J. G. Greene, "Loss of Enteric Dopaminergic Neurons and Associated Changes in Colon Mortility in an MPTP Mouse Model of Parkinson's Disease," Experimental Neurology, Vol. 207, No. 1, 2007, pp. 4-12. doi:10.1016/j.expneurol.2007.05.010

[12] G. Natale, O. Kastsiushenka, F. Fulceri, S. Ruggieri, A. Paparelli and F. Fornai, "MPTP-Induced Parkinsonism Extends to a Subclass of TH-Positive Neurons in the Gut," Brain Research, Vol. 1355, No. 8, 2010, pp. 195-206. doi:10.1016/j.brainres.2010.07.076

[13] A. G. Kanthasamy, M. Kitazawa, A. Kanthasamy and V. Anantharaman, "Dieldrin-Induced Neuro Toxicity: Relevance to Parkinson's Disease Pathogenesis," Neurotoxicology, Vol. 26, No. 4, 2005, pp. 701-719.

[14] S. A. Lloyd, C. Faherty and R. J. Smeyne, "Adult and in Utero Exposure to Cocaine Alters Sensitivity to the Parkinsonian Toxin 1-Methyl-4-phenyl-1,2,3,6-tetrahy-dropyridine," Neuroscience, Vol. 137, No. 3, 2006, pp. 905-913. doi:10.1016/j.neuroscience.2005.09.035

[15] C. G. Charlton, "A Parallel Relationship between Parkinson's Disease and Excess of S-Adenosylmethionine-dependent Biological Methylation in the Brain," Basic, Clinical and Therapeutic Aspects of Alzheimer's and Parkinson's 
Disease, Vol. 1, Cpt. 65, 1990, pp. 333-339.

[16] C. G. Charlton and B. Crowell, "Parkinson's Disease-Like Effects of S-Adenosyl-methionine: Effects of L-dopa," Pharmacology Biochemistry and Behavior, Vol. 43, No. 2, 1992, pp. 423-431. doi:10.1016/0091-3057(92)90172-C

[17] L. Axelrod and J. Tomchick. "Enzymatic-O-methylation of Epinephrine and Other Catechols," Journal of Biological Chemistry, Vol. 233, No. 3, 1958, pp. 702-705.

[18] C. G. Charlton and B. Crowell, "Striatal Dopamine Depletion, Tremors and Hypokinesia Following the Intracranial Injection of S-Adenosylmethionine," Molecular and Chemical Neuropathology, Vol. 26, No. 3, 1995, pp. 269284. doi:10.1007/BF02815143

[19] C. G. Charlton and J. Mack, "Substantia Nigra Degeneration and Tyrosine Hydroxylase Depletion Caused by Ex- cess S-Adenosylmethionine in the Rat Brain: Support for an Excess Methylation Hypothesis for Parkinsonism," Molecular Neurobiology, Vol. 9, No. 1-3, 1994, pp. 149-161. doi:10.1007/BF02816115

[20] G. Muthian, V. Mackey and C. G. Charlton, "Modeling a Sensitization Stage and a Precipitation Stage for Parkinson's Disease Using Prenatal and Postnatal 1-Methyl-4phenyl-1,2,3,6-tetrahydropyridine (MPTP) Administration," Neuroscience, Vol. 169, No. 3, 2010, pp. 1085-1093. doi:10.1016/j.neuroscience.2010.04.080

[21] C. G. Charlton, "Depletion of Nigrostriatal and Forebrain Tyrosine Hydroxylase by S-Adenosylmethionine: A Model That May Explain the Occurrence of Depression in Parkinson' Disease," Life Sciences, Vol. 61, No. 5, 1997, pp. 495-502. doi:10.1016/S0024-3205(97)00409-8

\begin{abstract}
Abbreviations
CNS, Central Nervous System; DA, Dopamine; DOPAC, 3,4-Dihydroxyphenylacetic acid; EC, Electro-chemical Detection; 5-HIAA, 5-Hydroxyindole acetic acid; 5-HT, 5-Hydroxytryptamine; HVA, Homo-vanillic acid; HPLC, High Performance Liquid Chromato-graphy; LPS, Lipopolysaccharide; LAAD, L-aromatic amino acid decarboxylase; MB, Midbrain; $\mathrm{MPP}^{+}$, 1-Methyl-4-phenylpyridnium; MPTP, 1-Methyl-4-phenyl -1,2,3,6-tetrahydropyridine; 3-MT, 3-Methoxytyramine; NS, Nigrostriatal; PBS, Phosphate Buffered Saline; PD, Parkinson's disease; TCA, Trichloro acetic acid; TH, Tyrosine hydroxyllase.
\end{abstract}

